\title{
The Astrological Influence on the Incidence of Hospital Admission and Length of Stay in Surgical Unit of a Regional Hospital
}

\author{
A. Chandio, A. Ali, A. Hussain, S. Fareed, M. Jalbani, F. Aftab, S. A. Naqvi \\ Department of Surgery, Mid Western Regional Hospital, Ennis, Republic of Ireland \\ Email: chandioashfaq@yahoo.com
}

Received 10 April 2014; revised 17 May 2014; accepted 21 June 2014

Copyright (C) 2014 by authors and OALib.

This work is licensed under the Creative Commons Attribution International License (CC BY). http://creativecommons.org/licenses/by/4.0/

(c) (i) Open Access

\section{Abstract}

Introduction: Though there is no scientific proof, some physicians and medical staff believe in association or influences of the zodiac signs on health. It was quite interesting to note that Kudva et al. from India found few definite attributable dental morphological traits and health to the specific sun signs and numerology. Salib from UK describes the distribution of suicide by hanging appeared significantly higher in those with a birth sign of Virgo and the lowest in Sagittarius and Scorpio. Aim: The aim of our study is to evaluate if there are any statistically significant documented frequencies of hospital admission emergency and elective for particular zodiac sign at all, and if they can be linked to astrological phenomena. Patients and Methods: Retrospective review of records of all the patients with hospital admission in a general surgical unit has been collected from July 2003 to June 2005. Demographic and clinical data were collected specifically Star signs and its relationship. Appropriate statistical analyses were performed. Results: In a period of 2 years, 1209 patients were admitted under one general surgeon of which 675 patients were male and 552 were female. Aries males and Cancer females had the most elective admissions while Libra females and Sagittarius males had minimum number of elective admissions. Aquarius male and Scorpio females had the most emergency admissions while Aquarius female and Scorpio male had the least emergency admissions. In elective setting, Pisces female and Aquarius male had minimum hospital length of stay (LOS) while both Cancer male and female had maximum LOS. In the emergency setting, both Cancer male and female had the minimum LOS while Pisces male and female had the maximum LOS. The incidence of acute appendicitis was the highest in Pisces at 19.39\%. Conclusion: Our retrospective data analysis documented some significant influence on hospital admission and length of stay under the influence of the star signs. This study sample was only General Surgical patients which exclude the number of other surgical and medical specialties. To achieve a real sample we need data of all the patients coming to the hospital.

\section{Keywords}

Astrological Influence

How to cite this paper: Chandio, A., Ali, A., Hussain, A., Fareed, S., Jalbani, M., Aftab, F. and Naqvi, S.A. (2014) The Astrological Influence on the Incidence of Hospital Admission and Length of Stay in Surgical Unit of a Regional Hospital. Open Access Library Journal, 1: e524. http://dx.doi.org/10.4236/oalib.1100524 


\section{Introduction}

Indo-European cultures developed a zodiac of twelve signs associated with a yearly cycle and with constellations of stars that lie along the apparent path of the Sun. The term zodiac meaning "circle of little animals", denotes several places where a circle of twelve animals occurs. The influence of the zodiac signs on health is not well-known. The aim of our study is to evaluate the influence of zodiac sign on the hospital admission and duration of inpatient stay. A review of the literature did not show any studies similar to the one we did; even though a number of studies related to lunar phase were found.

\section{Patients and Methods}

We conducted retrospective review of records of all the patients admitted under the care of a single consultant general/vascular surgeon over a two-year period from July 2003 to June 2005. Demographic and clinical data including zodiac signs were collected. Data were recorded onto a spread sheet (Microsoft[r] Excel 2002, Seattle, Washington, USA) conceding different ways of analysis and tabulations and appropriate statistical analysis was performed on SPSS (Version 14).

\section{Results}

1209 patients were admitted during the study period. There were 675 male and 552 female patients. Aries males and Cancer females had the highest number of elective admissions while Libra females and Sagittarius males had least admissions. In elective setting Pisces females and Aquarius males had minimum hospital length of stay (LOS) as compared to Cancer males and females had maximum LOS. In Emergency admissions Cancer males and females had the minimum LOS while Pisces males and females had the maximum LOS. Aquarius males and Scorpio females had the highest emergency admissions while Aquarius females and Scorpio males had the least emergency admissions. The incidence of acute appendicitis was highest in Pisces at 19.39\%. Table 1, Figure 1 \& Figure 2 summarize the results. The most number of trauma admissions where Gemini.

Table 1. Show the zodiacs and system involved.

\begin{tabular}{cccccccccccc}
\hline & App. & Hepato. & Hernia & Lower GI & Miscellaneous & Trauma & Upper GI & Vascular & Total & Most & Percentage \\
\hline Aries & 14 & $\mathbf{1 6}$ & 3 & 15 & 29 & 14 & $\mathbf{2 3}$ & 9 & 123 & Upper GI & 18.70 \\
Taurus & 4 & 10 & $\mathbf{9}$ & 15 & 29 & 7 & 11 & $\mathbf{1 8}$ & 103 & Vascular & 17.48 \\
Gemini & 8 & 7 & 6 & 13 & 21 & $\mathbf{1 9}$ & $\mathbf{2 2}$ & 11 & 107 & Upper GI & 20.56 \\
Cancer & 9 & 15 & 4 & 17 & 31 & 9 & 15 & 12 & 112 & Lower GI & 15.18 \\
Leo & 8 & 13 & 5 & 14 & 22 & 13 & 13 & 10 & 98 & Lower GI & 14.29 \\
Virgo & 16 & 9 & 7 & 10 & 27 & 9 & 16 & 8 & 102 & Upper Gi/App & 15.69 \\
Libra & 9 & 5 & 4 & 14 & 31 & 8 & 12 & 5 & 88 & Lower GI & 15.91 \\
Scorpio & 4 & 15 & 6 & 16 & 24 & 9 & 13 & 11 & 98 & Lower GI & 16.33 \\
Sagittarius & 14 & 11 & 3 & 9 & 22 & 9 & 7 & 12 & 87 & Appendicitis & 16.09 \\
Capricorn & 8 & 11 & 8 & $\mathbf{2 1}$ & 16 & 10 & 11 & 5 & 90 & Lower GI & 23.33 \\
Aquarius & 9 & 11 & 2 & 15 & 32 & 9 & 19 & 6 & 103 & GU/Upper GI & 18.45 \\
Pisces & $\mathbf{1 9}$ & 8 & 4 & 11 & 30 & 8 & 13 & 5 & 98 & Appendicitis & 19.39 \\
Total & 122 & 131 & 61 & 170 & 314 & 124 & 175 & 112 & 1209 & Upper GI & 14.47 \\
\hline
\end{tabular}

Note: Miscellaneous includes GU, Musculoskeletal, non-specific abdominal pain, respiratory problems, skin lesions, cellulites and infections. 


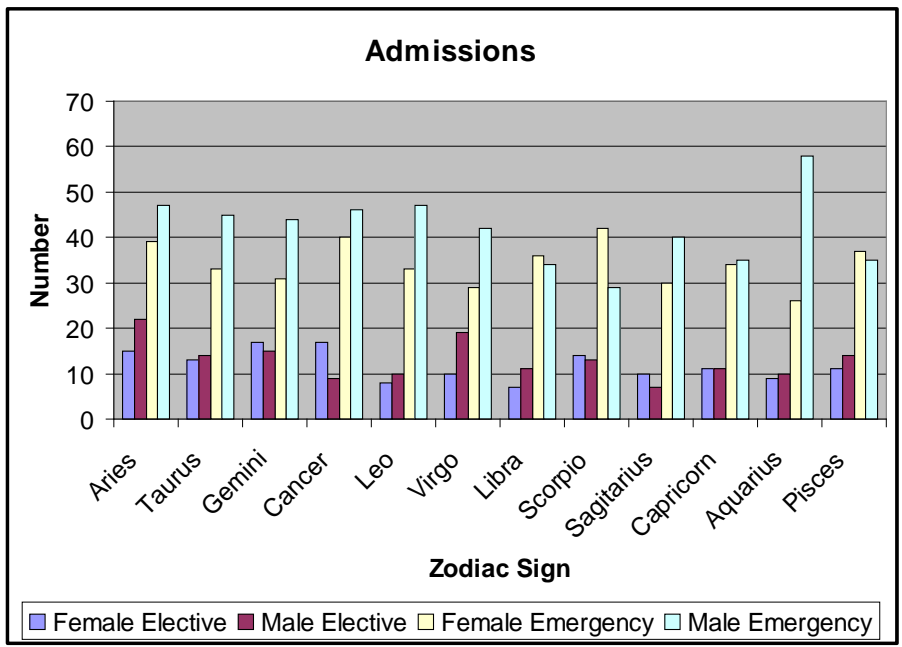

Figure 1. Total number of admission of different zodiacs.

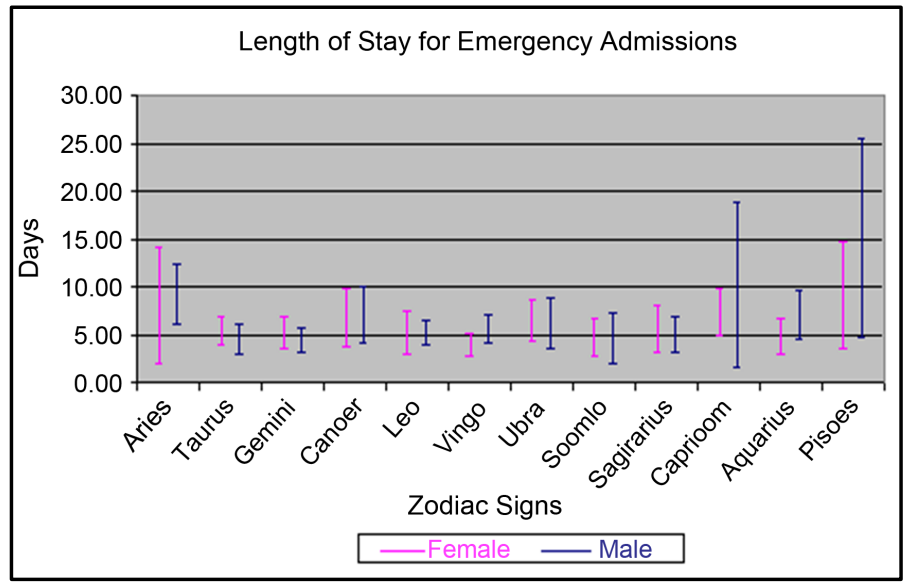

Figure 2. Length of stay for emergency admission of different zodiacs.

\section{Discussion}

As the claims of astrology are impossible to test in controlled laboratory conditions and cannot be reproduced, Astrology is no longer regarded as a science by many. However, the majority of those who read their "star signs" can identify aspects of their personality in what they read and it is possible that this may influence their attitudes and actions [1]. There have been numerous studies related to the influence of the lunar phases and health of mankind. Studies have not been able to pinpoint a correlation of trauma and lunar phase but have suggested that more studies are needed to asses the influence [2]. Dental morphological traits and health have been linked to the specific star signs and numerology of patients in a study by Kudva et al. [3]. Studies on psychiatric patients and the rate of suicides have found that the incidence of suicide by hanging is highest in the birth sign of Virgo and lowest in Sagittarius and Scorpio [1]. The most negativistic sign of Pisces was significantly associated with suicide ideation in another study by Stack et al. [4]. The belief that supernatural forces influence man's fate appears to be increasing. Many people are convinced that unknown forces can cause injuries.

\section{Conclusion}

Our study shows notable variation on hospital admission and length of stay based on different star signs. The study only targeted General Surgical admission and can be done for other medical specialties as well. The influence of star signs on other fields of medicine remains unknown. The clinical significance and application of these findings are debatable and further studies may be conducted to establish a correlation. 


\section{References}

[1] Salib, E. (2003) Astrological Birth Signs in Suicide: Hypothesis or Speculation? Medicine Science and the Law, 43, 111-114. http://dx.doi.org/10.1258/rsmmsl.43.2.111

[2] Zargar, M., Khaji, A., Kaviani, A., Karbakhsh, M., Yunesian, M. and Abdollahi, M. (2004) The Full Moon and Admission to Emergency Rooms. Indian Journal of Medical Sciences, 58, 191-195.

[3] Kudva, S. and Bhat, A.P. (2000) Teeth and Numerology from Zodiac Signs. A Correlative Study. Indian Journal of Dental Research, 11, 71-74.

[4] Stack, S. and Lester, D. (1988) Born under a Bad Sign? Astrological Sign and Suicide Ideation. Perceptual and Motor Skills, 66, 461-462. http://dx.doi.org/10.2466/pms.1988.66.2.461 